\title{
Molecular Docking Studies of Human Ubiquitin Conjugating Enzyme RAD6: A Systematic in Silico Approach
}

\author{
Dhafir Latief Fayadh ${ }^{1}$, Dr. K. Venkateswara Swamy ${ }^{2}$ \\ ${ }^{1}$ Department of Biotechnology, Fergusson College, University of Pune \\ ${ }^{2}$ Bioinformatics Laboratory, Dr. D.Y.Patil Biotechnology and Bioinformatics Institute, Dr. D. Y.Patil Vidyapeeth, Tathawade Campus, \\ Tathawade, Pune, India
}

\begin{abstract}
This study confirms the ubiquitin conjugating enzyme $2 B$ (Rad6) plays a significant role in the DNA repair pathway also because the ubiquitin-conjugating pathway. The DNA repair pathway could be a variety of bypass repair mechanism where the broken base pair is bypassed by permitting the replication fork to labor under the site of injury. This is often done by a shift mechanism wherever deoxyribonucleic acid enzyme - $\delta$ is switched with DNA enzyme - $\eta(D N A P-\eta)$. Site of DNAP - $\eta$ is massive enough to permit the broken ester to labor under, and so bypass the broken nucleotide. However, this is often potential solely through the involvement of Proliferating cell nuclear antigen (PCNA) that could be a processivity issue and it acts as a platform for the achievement of DNAP - $\eta$. Once the DNAP - $\eta$ is recruited, the DNA bypass mechanism is initiated. PCNA is activated by ubiquitination of essential amino acid residue by Rad6-Rad18 advanced. Once Rad6 is ubiquitylated, it forms complex with Rad18 and this complex then ubiquitylated PCNA that successively initiates error-free DNA bypass repair. Typically, attributable to exposure to radiation the Rad6-Rad18 advanced is not shaped. Within the absence of Rad6-Rad18 advanced, PCNA isn't activated and DNAP - $\eta$ isn't recruited at the harm the site. Therefore, deoxyribonucleic acid bypass mechanism isn't initiated. We tend to intend the activation of Rad6 by the triazole compounds to make a complex with Rad18 and ubiquitination of PCNA to initiate deoxyribonucleic acid bypass repair.
\end{abstract}

Keywords: molecular docking, human ubiquitin conjugating enzyme (Rad6), in silico

\section{Introduction}

\subsection{Cancer}

Cancer may be a category of diseases in which a bunch of cells show uncontrolled growth, invasion that intrudes upon and destroys adjacent tissues, and generally metastasis, or spreading to different locations within the body via lymph or blood. These 3 malignant properties of cancers differentiate them from benign tumors, that don't invade or spread. Cancer is primarily an environmental disease, although genetics influences the danger of some cancers [1]. Common environmental factors resulting in cancer include: tobacco, diet, obesity, infections, radiation, lack of physical activity, and environmental pollutants. These environmental factors cause or enhance abnormalities within the genetic material of cells. Cell reproduction is an especially complicated method that's commonly tightly regulated by many categories of genes, together with oncogenes and neoplasm suppressor genes. Hereditary or non-inheritable abnormalities in these regulative genes will cause the event of cancer.

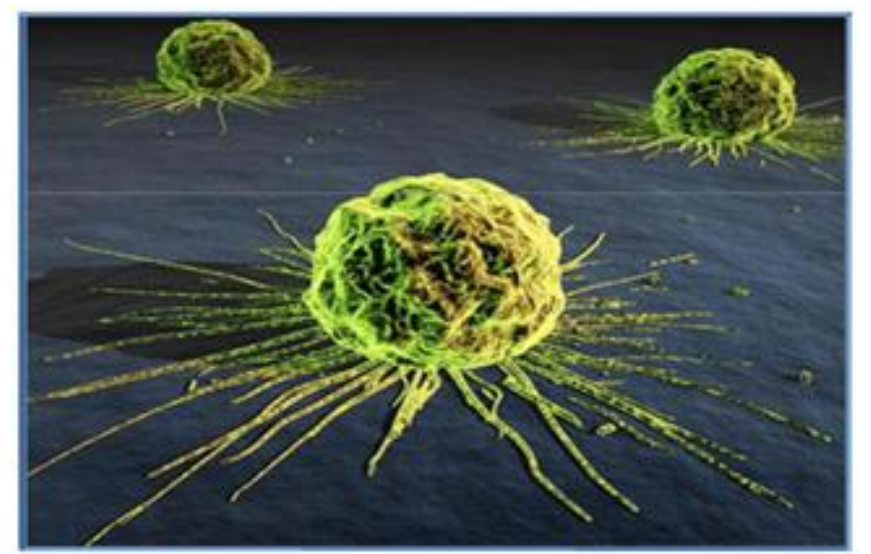

Figure 1: Graphical representation of cancer

\subsection{DNA Damage}

DNA damage, attributable to environmental factors and traditional metabolic processes within the cell, happens at a rate of 1,000 to $1,000,000$ molecular lesions per cell per day [3]. Whereas this constitutes solely $0.000165 \%$ of the human genome's more or less 6 billion bases ( 3 billion base pairs), unrepaired lesions in crucial genes (such as neoplasm suppressor genes) will impede a cell's ability to carry outitsfunction and appreciably increase the chance of tumor formation. The overwhelming majority of DNA harm affects the first structure of the double helix; that's, the bases themselves are chemically modified.

\subsection{Sources of DNA damage}

DNA damage are often divided into 2 main types:

- Endogenous damage such as attack by reactive oxygen species produced from normal metabolic by-products 


\section{International Journal of Science and Research (IJSR) \\ ISSN (Online): 2319-7064}

Index Copernicus Value (2013): 6.14 | Impact Factor (2014): 5.611

(spontaneous mutation), especially the process of oxidative deamination and also includes replication errors.

- Exogenous damage caused by external agents such as,

i. Ultraviolet (UV 200-300nm) radiation from the sun

ii. Other radiation frequencies, including x-rays and gamma rays iii. Hydrolysis or thermal disruption

iii. Certain plant toxins

iv. Human-made mutagenic chemicals, especially aromatic compounds that act as DNA intercalating agents

v. Cancer chemotherapy and radiotherapy

vi. Viruses

The replication of damaged DNA before cell division can lead to the incorporation of wrong bases opposite damaged ones. Daughter cells that inherit these wrong bases carry mutations from which the original DNA sequence is unrecoverable (except in the rare case of a back mutation, for example, through gene conversion).

\subsection{Types of DNA Damage}

There are five main types of damage to DNA due to endogenous cellular processes:

1) Oxidization of bases and generation of DNA strand interruptions from reactive oxygen species.

2) Alkylation of bases like formation of 7-methylguanine, 1-methyladenine, 6-O-Methylguanine.

3) Hydrolysis of bases, like chemical processes, deprivation, and depyrimidination.

4) Large Adduct Formation

5) Mismatch of bases, attributable to errors in DNA replication, during which the incorrect DNA base is stitched into place in a freshly forming DNA strand, or a DNA base is skipped or erroneously inserted.

\subsection{DNA repair and cancer}

Inherited mutations that have an effect on DNA repair genes are powerfully related to high cancer risks in humans. Hereditary non-polyposis colorectal cancer (HNPCC) is powerfully related to specific mutations within the DNA mismatch repair pathway. BRCA1 and BRCA2, 2 famous mutations conferring a vastly increased risk of breast cancer on carriers, are each related to large number of DNA repair pathways, particularly NHEJ and homologous recombination.

Cancer therapy procedures such as chemotherapy and radiotherapy work by overwhelming the capacity of the cell to repair DNA damage, resulting in cell death. Cells that are most rapidly dividing - most typically cancer cells - are preferentially affected. The side-effect is that other noncancerous but rapidly dividing cells such as stem cells in the bone marrow are also affected. Modern cancer treatments attempt to localize the DNA damage to cells and tissues only associated with cancer, either by physical means (concentrating the therapeutic agent in the region of the tumor) or by biochemical means (exploiting a feature unique to cancer cells in the body).

\section{Aim and Objective}

Aim: Molecular docking studies of Human Ubiquitin Conjugating EnzymeRad6: A systematic in silico approach.

Objective: The ubiquitin conjugating protein 2B (Rad6) plays a serious role within the DNA repair pathway as well as the ubiquitin-conjugating pathway. The DNA repair pathway may be a variety of bypass repair mechanism wherever the broken nucleotide is bypassed by permitting the replication fork to pass through the site of damage. This is often done by a shift mechanism wherever DNA Polymerase $-\delta$ is switched with DNA Polymerase - $\eta$ (DNAP - $\eta$ ). Active site of DNAP - $\eta$ is large enough to allow the damaged nucleotide to pass through and thus bypass the damaged base pair. However, this is often attainable solely through the involvement of Proliferating Nuclear Cell Antigen (PCNA) which is a processivity factor and it acts as a platform for the recruitment of DNAP - $\eta$. Once the DNAP - $\eta$ is recruited, the DNA bypass mechanism can be initiated. PCNA is activated by ubiquitylation of Lysine residue by Rad6-Rad18 complex. When Rad6 is ubiquitylated, it forms a complex with Rad18 and this complex then ubiquitylates PCNA, which in turn initiates error-free DNA bypass repair. Generally, due to exposure to radiation the Rad6-Rad18 complex is not formed. In the absence of Rad6-Rad18 complex, PCNA is not activated and DNAP - $\eta$ is not recruited at the damage site. Thus DNA bypass mechanism is not initiated.

In our work, we have taken the protein structure of human Rad6 (hRad6) and Docking studies were carried out using Auto dock vina in silico, to understand the behavior of hRad6. A library of eleven triazole compounds were generated which were docked individually to hRad6 to observe the binding affinity of the compounds. We intend the activation of Rad6 by the triazole compounds to form a complex with Rad18 and ubiquitination of PCNA to initiate DNA bypass repair.

\section{Literature Survey}

\subsection{Ubiquitin}

Ubiquitin could be a little regulative supermolecule that has been found in most tissues (ubiquitously) of organism organisms. Among alternative functions, it directs proteins utilization.

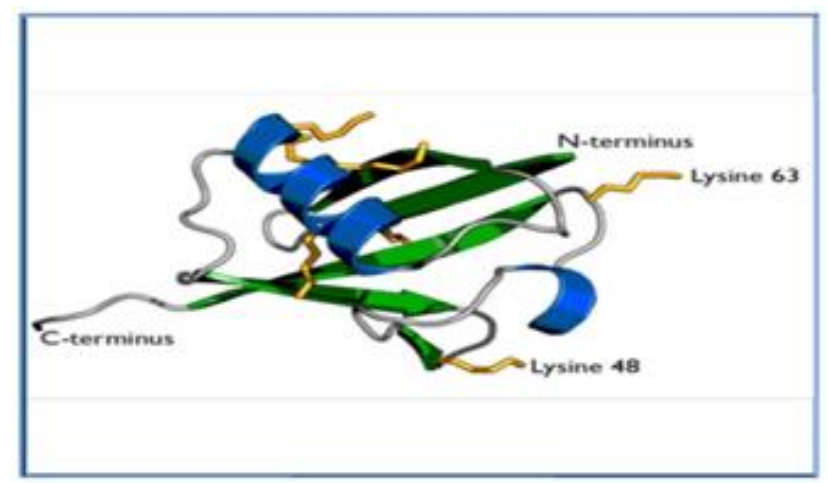

Figure 2: Ubiquitin protein 


\section{International Journal of Science and Research (IJSR) \\ ISSN (Online): 2319-7064}

Index Copernicus Value (2013): 6.14 | Impact Factor (2014): 5.611

Ubiquitin could be a little super molecule that exists all told organism cells. It performs its myriad functions through conjugation to an outsized vary of target proteins. A spread of various modifications will occur. The ubiquitin super molecule itself consists of seventy-six amino acids and includes a molecular mass of concerning eight. $5 \mathrm{kDa}$. Key options embrace its C-terminal tail and therefore the seven lysine residues. It's extremely preserved among organism species: Human and yeast ubiquitin share ninety-six sequence identity. Ubiquitin binds to proteins and labels them for destruction. The ubiquitin tag directs proteins to the proteasome that is a cell organ within the cell that degrades and recycles inessential proteins. Ubiquitin tags can even direct proteins to alternative locations within the cell, where they management alternative super molecule and cell mechanisms.

\subsection{Ubiquitin-conjugating accelerator}

Ubiquitin-conjugating enzymes, additionally called E2 enzymes and a lot of seldom as ubiquitin-carrier enzymes, perform the second step in theubiquitinationreaction that targets a protein for degradation via the proteasome. The ubiquitination method covalently attaches ubiquitin, a brief super molecule of seventy-six amino acids, to a lysine residue on the target super molecule. Once a super molecule has been labelled with one ubiquitin molecule, extra rounds of ubiquitination type a polyubiquitin chain that's recognized by the proteasome's $19 \mathrm{~S}$ restrictive particle, triggering the ATP-dependent evolution of the target super molecule that permits passage into the proteasome's $20 \mathrm{~S}$ core particle, wherever proteases degrade the target into short amide fragments for use by the cell.

\subsection{DNA harm bypass}

All organisms got to subsume the issues that arise once a moving replication fork encounters harm within the model strand [10]. Clearly the most effective thanks to subsume this example is to repair the harm by $\mathrm{AN}$ excision mechanism. In some cases, however, the harm might not be fixable, or the advancing replication fork could have already got unwound the parental strands, therefore preventing excision mechanisms from victimization the complementary strand as model for repair, or excision repair might not nevertheless have had a chance to repair the harm.

There square measure 2 reasons why it's necessary for the cell to be able to move replication forks past unrepaired harm,

1) long-run blockage of replication forks results in necrobiosis.

2) Replication of broken deoxyribonucleic acid provides a sister fibril that may be used as model for future repair by homologous recombination.

Notice that replication fork bypass mechanisms cannot, to be precise, be thought-about samples of DNA repair, as a result of the harm is left within the DNA, a minimum of briefly. Nonetheless, experiments in yeast demonstrate that harm bypass is a very important element of the cellular response to DNA harm. It contributes to cellular survival of radiation harm to roughly identical extent because the pathways for ester excision repair and repair by homologous recombination.

Eukaryotic mechanisms for replication fork bypass of broken sites in DNA aren't moreover understood, however significant progress has been created in recent years. Moreover, currently that the excision repair pathways are for the most part puzzled out, several scientists inquisitive about cellular responses to DNA harm square measure turning their attention to replication bypass.

\subsection{Re-combinational harm bypass}

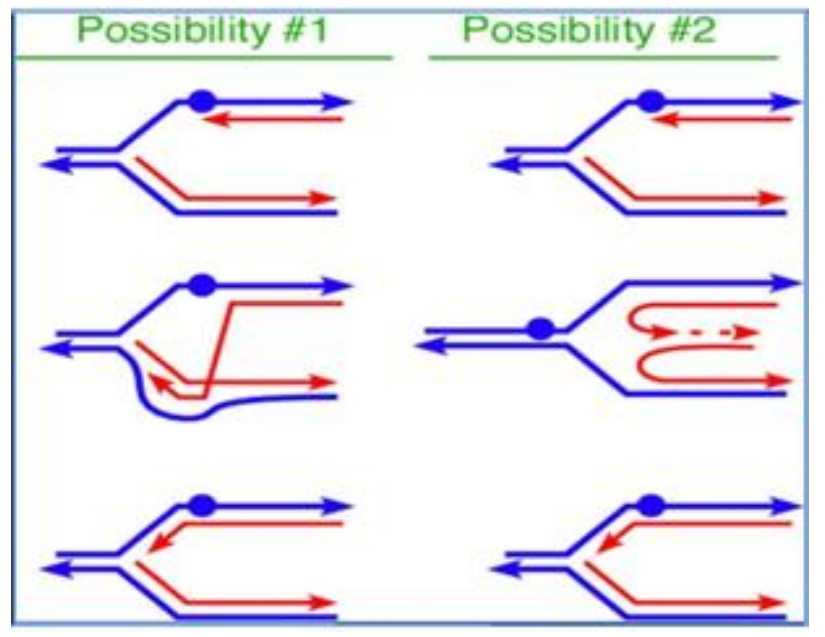

Figure 3: Possibilities for Re-combinational bypass

The mechanism of organism Re-combinational bypass isn't yet clear. Two potentialities square measure shown in Figure three. In each of those potentialities, synthesis of 1 red emergent strand (the leading strand) is blocked by DNA harm (closed circles). Synthesis of the opposite red emergent strand (the insulation strand) continues for a distance on the far side the broken region. This can be necessary, as a result of this complementary emergent strand can give a model for Re-combinational bypass synthesis through the broken region. Within the left-hand example, the blocked leading strand enzyme is alleged to switch to use of the emergent sister strand as model. Comfortable leading strand DNA is synthesized victimization this sister strand model to bypass the harm. Then the leading strand is straight from its sister strand model, and therefore the enzyme switches back to victimization the highest parental strand as model.

\section{Research Methodology}

To get an improved understanding of the situation of hRad6 we have a tendency to search model 2YB6 in PDBsum server. PDBsum provides a summary of each molecule structure deposited within the super molecule knowledge Bank (PDB), giving schematic diagrams of the molecules in every structure and therefore the interactions between them (Figure 4). From PDBsum we discover out the preserved residues of 2 YB6 


\section{International Journal of Science and Research (IJSR) \\ ISSN (Online): 2319-7064}

Index Copernicus Value (2013): 6.14 | Impact Factor (2014): 5.611

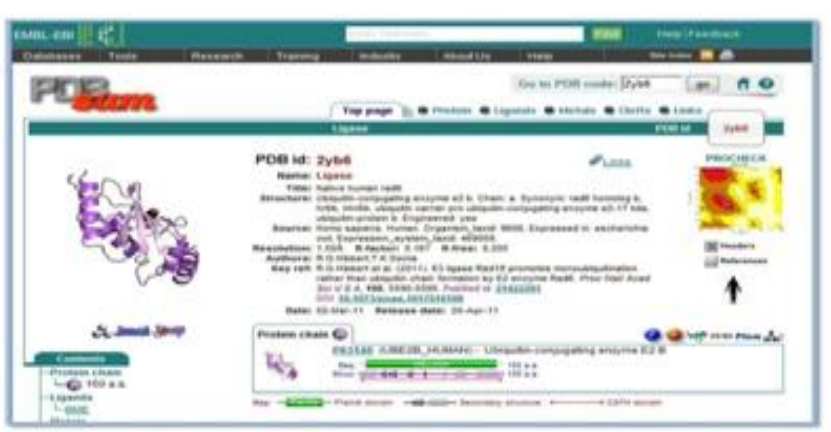

Figure 4: PDBsum entry for 2YB6

\subsection{Calculation of molecular properties of ligands}

The molecular properties and drug likeness of the triazole compounds were calculated. The drug likelihood was evaluated supported Lipinski's Rule of 5.

Lipinski's Rule of 5 is employed to judge drug likeness, or verify if a chemical compound with an exact pharmacologic or biological activity has properties that will build it a probable orally active drug in humans. The rule was developed by Christopher A. Lipinski in 1997. Supported the observation that almost all medication medicine square measure comparatively little and solvophilic molecules. The rule describes molecular properties necessary for a drug's pharmacology within the bod, together with their absorption, distribution, metabolism, and excretion ("ADME").

\subsection{Molecular docking studies}

In the field of molecular modeling, moorage could be a methodology that predicts the well-liked orientation of 1 molecule to a second once certain to one another to create a stable advanced. Information of the well-liked orientation successively is also wont to predict the strength of association or binding affinity between 2 molecules victimization for instance rating functions. The associations between biologically relevant molecules like proteins, nucleic acids, carbohydrates, and lipids play a central role in signal transduction. Moreover, the relative orientation of the 2 interacting partners could have an effect on the kind of signal made. Thus moorage is beneficial for predicting each the strength and sort of signal made.

Docking is usually wont to predict the binding orientation of little molecule drug candidates to their super molecule targets so as to successively predict the affinity and activity of the tiny molecule. Therefore, moorage plays a very important role within the rational style of medication.

In this report versatile moorage methodology is employed with the assistance of motor vehicle dock vina and MGL TOOLS. Versatile moorage procedures allow conformational modification within the bond angles, bond lengths and torsional angles of the ligands moreover as enable facet chains of the super molecule target to stay versatile.

\section{Results and Discussion}

\subsection{Active Site Analysis}

The active site of hRad6 was analyzed by studying its template 2YB6 in PDBsum (Figure 5).

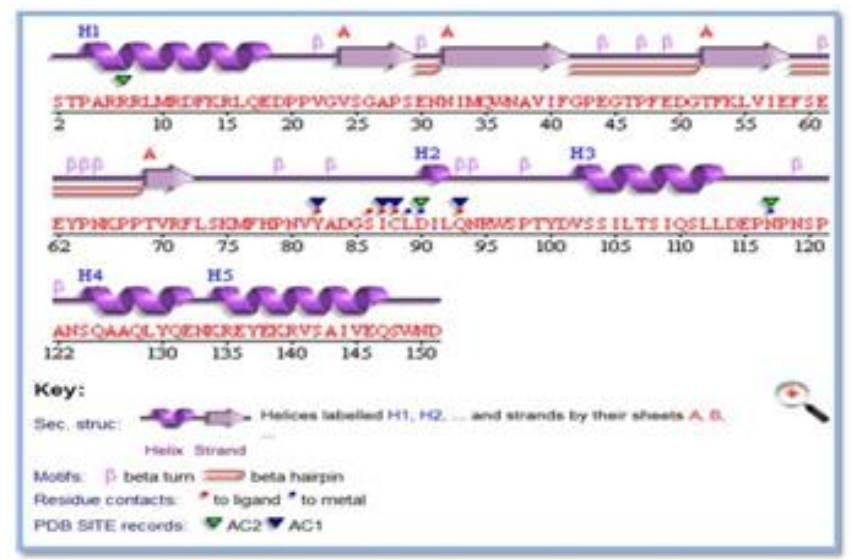

Figure 5: PDBsum entry for 2YB6 describing the conserved residues

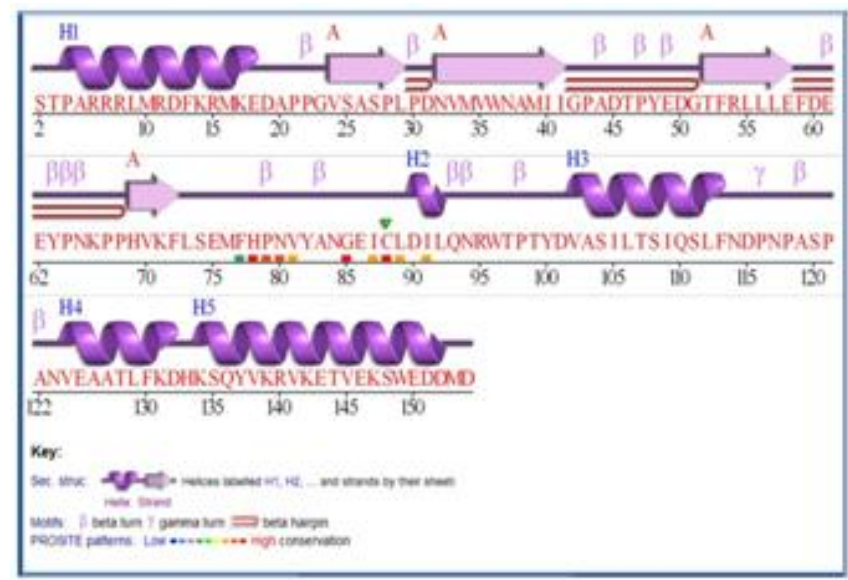

Figure 6: PDBsum entry for S. cerevisiae Rad6 (PDB ID: $1 \mathrm{AYZ}$

From the analysis of the PDBsum entry for 2YB6 (Figure six.1.1) it had been discovered that residues that lie inside the vary of Tyr eighty-two TO GLN ninety-threeare showing high conservation. Also, CYS88 lies during this region of high conservation. Just in case of yeast Rad6 (PDB ID: $1 \mathrm{AYZ}$ ) it had been discovered that CYS88 is that the catalytic residue. (Figure 6).

\subsection{Calculation of molecular properties of ligands}

The molecular properties of the triazole compounds were calculated exploitation the MOLINSPIRATION server. All the eleven triazole compounds were drawn within the JME editor at the server and also the properties were calculated to get an improved understanding of those ligands in terms of their drug likeness. 


\section{International Journal of Science and Research (IJSR) \\ ISSN (Online): 2319-7064}

Index Copernicus Value (2013): 6.14 | Impact Factor (2014): 5.611

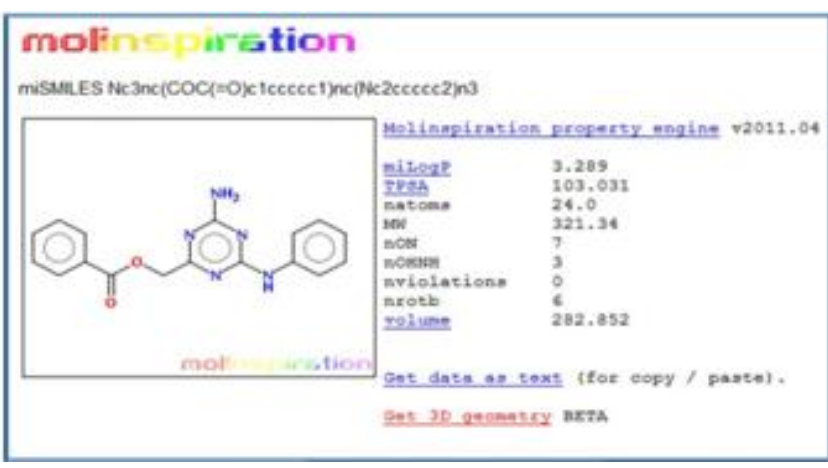

Figure 7: Molinspiration result page displaying the various properties calculated for a ligand

\subsection{Molecular tying up analysis}

After careful study of the molecular properties of the eleven triazole compounds, tying up studies were applied for all the eleven compounds with hRad6 exploitation Vina. The tying up results were then analyzed with reference to the separation energy, range of element bonds shaped, residues concerned in bond formation and bond distances of the element bonds shaped.

Binding Energy: The energy needed to separate a molecule/ligand from the macromolecule to that it's certain. Car dock vina calculate the separation energy because the total of unit energy and also the torsional free energy penalty. The lower the worth of the separation energy, stronger the matter is sure to the receptor.

Hydrogen Bonding: It plays a really vital role in matter binding to a target macromolecule. So the quantity of element bonds shaped, the residues concerned in bonds and element bond distances are necessary in evaluating the strength with that a matter would bind to its target macromolecule.

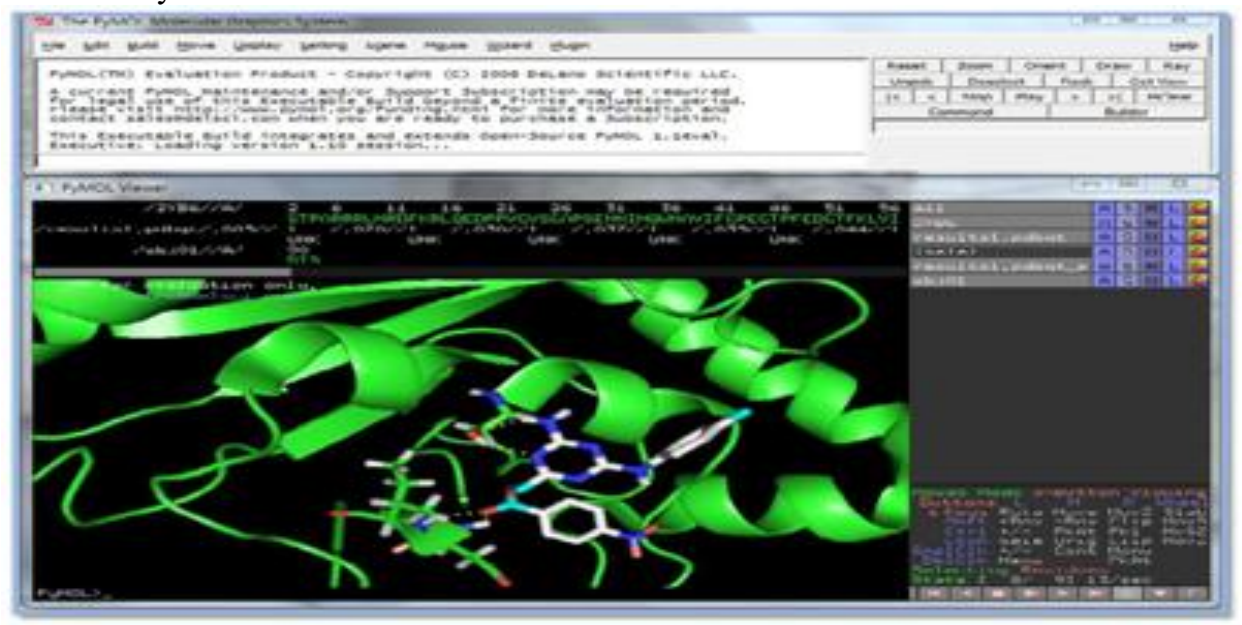

Figure 8: Analysis of the docking conformations in PyMOL

Images of triazole compounds docked with hrad6. The interacting residues are labeled, the hydrogen bonds are shown in yellow dotted line and their bond distances are also displayed

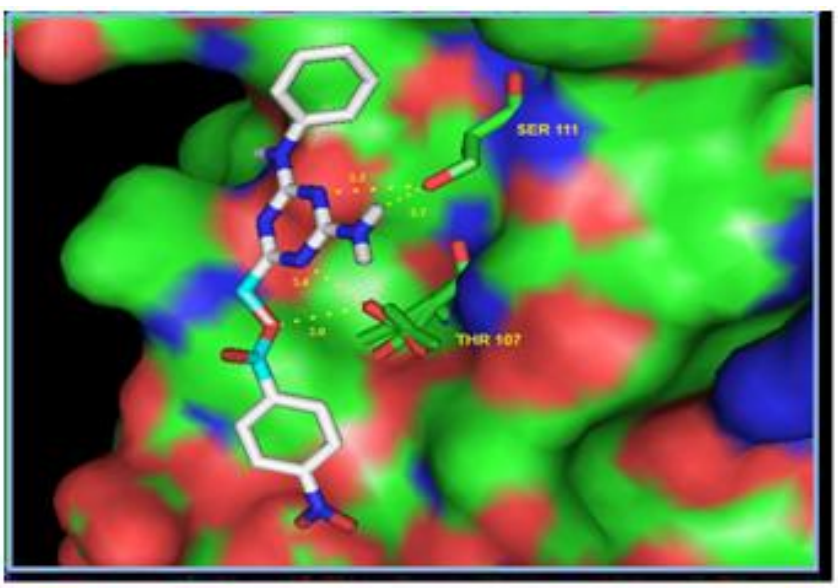

Figure 9: TZ002

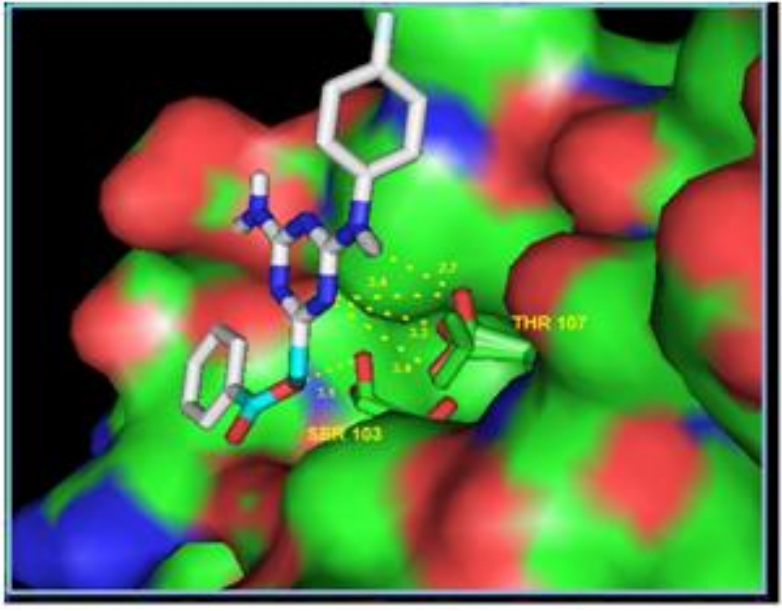

Figure 10: TZ011

\section{Conclusion}

The 3D structure of hRad6 was retrieved from macromolecule information Bank (PDB) pdb code (2YB6) and therefore the structure was then thought of for molecular tying up studies with eleven triazole (TZ) compounds. Situation of hRad6 was discovered by PDBsum analysis. 
2YB6 the tying up studies were performed victimization car dock vina. The properties of thetriazole compounds were calculated victimization MOLINSPIRATION and therefore the tying up studies unconcealed that TZ002 and TZ011 are the simplest ligands as compared to the others in terms of their energy, and range of element bonds fashioned between the substance and therefore the residues of hRad6. TZ002 has an overall energy of $-7.1 \mathrm{kcal} / \mathrm{mol}$, and forms four element bonds inside the situation of hRad6. TZ002 forms 2 chemical bond with SER 111, and 2 with THR 107. TZ011 has associate degree overall energy of $-6.8 \mathrm{kcal} / \mathrm{mol}$, and it forms 5 element bonds inside the situation of hRad6. TZ011 forms one chemical bond with SER 103 and 4 with THR 107. From the tying up studies it had been clear that TZ002 and TZ011 are probably to be lead compounds which will bind favorably to hRad6 and convey regarding the meant action that is activation of Rad6 to bind to $\operatorname{Rad} 18$ to create a Rad6-Rad18 advanced. This advanced will then activate PCNA, by ubiquitination, and convey regarding the initiation of polymer harm bypass repair. From this study we advise in vivo studies of hRad6 victimization the triazole compounds since they are found to allow smart binding behavior in us in-silico studies.

\section{References}

[1] Anand P., Kunnumakkara A. B. et al., "Cancer is a preventable disease that requires major lifestyle changes", Pharm. Res. 25 (9) (2008): 2097-2116.

[2] Jemal A.; Bray F., Center M. M., Ferlay J., Ward E., Forman D., "Global cancer statistics", CA: a cancer journal for clinicians 61 (2) (2011): 69-90.

[3] Lodish H., Berk A., Matsudaira P., Kaiser C. A., Krieger M., Scott M. P., Zipursky S. L., Darnell J., "Molecular Biology of the Cell", WH Freeman: New York, NY. 5th ed. (2004): 963.

[4] Braig M.; Schmitt C. A., "Oncogene-induced senescence: putting the brakes on tumor development", Cancer Res. 66 (6) (2006): 2881-2884.

[5] Campisi J., d'Adda di Fagagna F., "Cellular senescence: when bad things happen to good cells", Rev. Mol. Cell Biol. 8 (9) (2007): 729-740

[6] Waters L. S., Minesinger B. K., Wiltrout M. E., D'Souza S., Woodruff R. V., Walker G. C., "Eukaryotic translation polymerases and their roles and regulation in DNA damage tolerance". Microbiol. Mol. Biol. Rev. 73 (1) (2009): 134-154.

[7] Martin Karplus, J. Andrew McCammon, "Molecular dynamics simulations of biomolecules", Nature Structural Biology 9 (9) (2002):646-652.

[8] http://www.ch.embnet.org/MD_tutorial/pages/MD.Part1 .html

[9] http://asajj.roswellpark.org/huberman/dna_repair/bypass .html

[10] Koken M. H., Smit E. M., Jaspers-Dekker I., Oostra B. A., Hagemeijer A., Bootsma D., Hoeijmakers J. H., "Localization of two human homologs, HHR6A and HHR6B, of the yeast DNA repair gene RAD6 to chromosomes Xq24-q25 and 5q23-q31", Genomics 12 (3) (1992): 447-453. 